Pacific Journal of Mathematics

DETERMINING AN ANALYTIC FUNCTION FROM ITS 


\section{DETERMINING AN ANALYTIC FUNCTION FROM ITS DISTRIBUTION OF VALUES}

\section{Peter Waksman}

Let $f(x)$ be a real analytic function defined on a (possibly infinite and possibly closed) interval $(A, B)$. The frequency distribution of $f$ is defined to be

$$
\omega_{f}(y)=\text { Lebesgue measure }\{x \in(A, B) \mid f(x) \leq y\} .
$$

In this paper we consider the problem of determining $f$ given its distribution $\omega_{f}$. Since a trivial change of $f$ of the form $g(x)=f(a \pm x)$ will have the same distribution, we ask: does $\omega_{f}$ determine $f$ up to such trivial changes? A partial answer is given by

THEOREM. If $f$ is real analytic with distinct and non-degenerate critical values on a finite interval $[A, B]$ and if the values of $f$ at the endpoints are different from each other, and at least one is different from the value at any critical point on the interior of the interval, then $f$ is determined uniquely (up to trivial changes) by its frequency distribution on this interval. As a consequence we have.

COROLLARY. A real analytic function with distinct non-degenerate critical values is determined uniquely (up to trivial changes) by its frequency distribution on the interval between its minimum and maximum critical points.

The method of proof depends on studying the behavior of the frequency distribution near a critical value, which method fails if the value is repeated at different critical points or if the value is a degenerate critical value $\left(f(x)\right.$, where $f^{\prime}(x)=0$ and $\left.f^{\prime \prime}(x)=0\right)$; thus the assumptions which exclude such cases may only be necessary for the proof and not the result. That the assumption of distinct endpoint values is necessary is seen in the following examples.

The functions $f_{1}(x)=x^{2}$ on $[-1,1]$ and $f_{2}(x)=(x / 2)^{2}$ on $[0,2]$ both have the frequency distribution $\omega(y)=2 \sqrt{y}$. The critical value 0 is distinct and non-degenerate, but the theorem does not apply to $f_{1}$ because $f_{1}(-1)=f_{1}(1)$. In general an even function is not determined by its distribution on an interval symmetric about the origin. The function $f_{2}$ does satisfy the assumptions of the theorem and is (up to trivial changes) the only such function with distribution $2 \sqrt{y}$. It is possible that $f_{1}$ is the 
only 2-1 function with this distribution, and it is tempting to try to extend the theorem, replacing the endpoint condition with an assumption about the degree ( $n$, if $f$ is $n-1$ ) of the function.

Periodic functions defined on an interval of length equal to a multiple of their period are also not determined by their distributions. For example $\sin (k x)$ on $[0,2 \pi]$ has the same distribution for any integer $k$. Here the critical values are not distinct (for $k>1$ ) and the endpoint values coincide. Again one might hope to extend the theorem by adding an assumption about the degree of the function to be determined; thus it is possible that $\sin (k x)$ is the only analytic function of degree $2 k$ with the given distribution. However, as it stands, the theorem only applies when the interval is not of length equal to a multiple of the period, and even then only when the values of critical points in the interior of the interval are distinct and when the endpoint values are different from each other and different from the values at interior critical points. Thus for example $\sin (x)$ is determined uniquely (up to trivial changes) on the interval $[0,2 \pi+\varepsilon]$ where $0<|\varepsilon|<\pi$.

If an analytic function has repeated critical values but has distinct and non-critical values at the endpoints, the arguments can be modified easily to show the function is determined by its distribution. However, no quick modification will prove the result when the endpoint values are critical values; yet the result might still be true. Thus there is more work to be done on this topic; both for understanding periodic and symmetric cases, as well as for trying to remove the assumption about distinctness and nondegeneracy of the critical values. We do not consider here the case of an infinite interval; in all these cases a version of the theorem may still hold, but other ideas will be needed for its proof.

Motivation. There are general reasons for wanting to view an analytic function as equivalent to its distribution. For example one might want to compute in terms of the distribution rather than the function; or, in an applied context, one might be able to measure the distribution but not the function. The present work was motivated by the following problem in non-linear evolution equations:

If $u(x, t)$ satisfies a differential equation of the form $u_{t}=F(u)$ (where $F$ can be an operator involving higher derivatives) is the initial condition $u(x, 0)$ determined by the time averages

$$
A(t)=\int u(x, t) d x ?
$$


In the simplest case, $F$ is a smooth function of $u$, in which case one can proceed as follows: $A(t)$ determines the derivatives $A^{(n)}(0)$; under mild assumption $A^{(n)}(0)=\int u_{t t \cdots t}(x, 0) d x$. By repeated applications of the relation $u_{t}=F(u)$ this integral can be rewritten as

$$
\int\left(F(g) \frac{d}{d g}\right)^{n-1} F(g) d x \text { where } g(x)=u(x, 0) .
$$

Substituting $y=g(x)$ this becomes

$$
\int\left(F(y) \frac{d}{d y}\right)^{n-1} F(y) d \omega_{g}(y),
$$

and in this form the problem is to determine $g$ given the quantities

$$
A^{(n)}(0)=\int \psi_{n}(y) d \omega_{g}(y),
$$

where the $\psi_{n}(y)=(F(y)(d / d y))^{n-1} F(y)$ depend only on $F$.

If, for example, the uniform closure of the linear span of $\left\{\psi_{n}\right\}$ contains the continuous functions, then $\omega_{g}(y)$ is determined at its points of continuity. In this case the initial condition $g(x)=u(x, 0)$ is determined when $g$ is known to satisfy the hypotheses of the theorem.

The paper is organized as follows: In $\S 1$ we study the density $d \omega_{f} / d y$, and prove a preliminary lemma used in the proof of the theorem which comprises $\$ 2$. The case where one endpoint is not a critical point is considered first and then the case where both endpoints are critical points. Interrupting the proof, there is a short digression about the existence of a function with given distribution.

1. The density $d \omega_{f} / d y$. In this section we develop an expression for the density $d \omega_{f} / d y$, which is used later in the proof. Throughout in the following let $f$ be a non-constant analytic function defined on a closed real interval $[A, B]$.

LEMMA 1. For any measurable function $p(y)$ and non-constant analytic function $f$ defined on $[A, B]$

$$
\int_{A}^{B} p(f) d x=\int_{\text {image }(f)} p(y) 1_{f}(y) d y
$$

where $1_{f}(y) \equiv \sum_{x \in f^{-1}(y)} 1 /\left|f^{\prime}(x)\right|$.

REMARK. This is just the change of variables formula when we substitute $y=f(x)$; but that it makes sense when $f$ is not monotonic is called "integration over the fibre" and it holds in greater generality than stated here [1]. A direct proof when $f$ is algebraic is as follows. 
Proof.

$$
\int_{A}^{B} p(f) d x=\sum_{\substack{\text { intervals } J \\ \text { where } f \text { is } \\ \text { monotonic }}}\left(\int_{J} p(f) d x\right) .
$$

The sum is finite because $f$ has a finite number of critical points. Now, substituting $y=\left(\left.f\right|_{J}\right)(x)$,

$$
\int_{J} p(f) d x=\int_{\text {image }\left(\left.f\right|_{J}\right)} p(y) \frac{1}{\left|f^{\prime}\left(\left.f\right|_{J} ^{-1}(y)\right)\right|} d y .
$$

Set

$$
h_{f}^{j}(y)=\left\{\begin{array}{cl}
0 & \text { if } y \notin f(J) \\
\frac{1}{\left|f^{\prime}\left(\left.f\right|_{J} ^{-1}(y)\right)\right|} & \text { if } y \in f(J) .
\end{array}\right.
$$

Then we have

$$
\int_{A}^{B} p(f) d x=\sum \int_{J} p(y) h_{f}^{J}(y) d y=\int_{\text {image }(f)} p(y)\left(\sum_{J} h_{f}^{J}(y)\right) d y
$$

and we see that

$$
1_{f}(y)=\sum_{J} h_{f}^{J}(y)
$$

satisfies

$$
1_{f}(y)=\sum_{x \in f^{-1}(y)} \frac{1}{\left|f^{\prime}(x)\right|} .
$$

This proves the lemma.

Setting $p(y)=$ characteristic function of $\left(-\infty, y_{0}\right)$ :

(1.1) measure $\left\{x \mid f(x) \leq y_{0}\right\}=\int_{f(x) \leq y_{0}} d x=\int_{-\infty}^{y_{0}} 1_{f}(y) d y$.

Thus (a) $1_{f} \in L^{1}(\operatorname{image}(f))$, and (b) $d \omega_{f} / d y=1_{f}$. Also, from the definition of $1_{f}$ we see that (c) $1_{f}$ is analytic between poles which are the critical values of $f$. Now, (b) tells us that determining $1_{f}$ is equivalent to determining $\omega_{f}$, so our problem is equivalent to determining $f$ given $1_{f}$.

2. The Main Theorem. For a simple statement of the main theorem we use a non-standard definition of critical value:

Define: The critical values of $f$ are either

(1) The $f(x)$ where $f^{\prime}(x)=0$

or

(2) $f(A)$ and $f(B)$, when $f$ is defined on $[A, B]$. 
We say a critical value $y_{0}$ is repeated if there exists $x_{1} \neq x_{2}$ such that $f\left(x_{1}\right)=f\left(x_{2}\right)=y_{0}$ and either $f^{\prime}\left(x_{i}\right)=0$ or $x_{i} \in\{A, B\}(i=1,2)$ (i.e. $y_{0}$ is a critical value in more than one way). If the critical values are not repeated we say they are distinct. A critical value $y_{0}$ is called degenerate if there is an $x$ such that $f(x)=y_{0}, f^{\prime}(x)=0, f^{\prime \prime}(x)=0$; if there is no such $x, y_{0}$ is called a nondegenerate critical value.

THEOREM. If, on $[A, B], f$ is an analytic function with distinct non-degenerate critical values, then $1_{f}$ determines $f$.

REMARK. To "determine $f$ " means that, up to translating and reflecting the domain, $f$ is the unique analytic function defined or a finite interval, with distinct non-degenerate critical values, having this $1_{f}$. Throughout below work with a fixed but unknown such analytic function $f$.

Proof. Setting the upper limit $y_{0}=\infty$ in (1.1), we have

$$
\int_{-\infty}^{\infty} 1_{f} d y=\text { measure }(\operatorname{domain}(f)),
$$

so the length of the interval of definition of $f$ is known; so we may take $[A, B]$ to be any interval of this length.

Case 1. Assume $f^{\prime}(A) \neq 0$ or $f^{\prime}(B) \neq 0$. [A short proof of this case can be given; we give a slightly longer proof here in order to develop concepts used later.] Reflecting the domain through its midpoint if necessary, we may assume $f^{\prime}(A) \neq 0$. Claim: in this case $f(A)$ is a non-pole discontinuity of $1_{f}$.

Proof of Claim. Let $J_{1}, J_{2}, \ldots, J_{k}$ be the consecutive intervals on which $f$ is monotonic ( $\operatorname{so} \inf \left(J_{1}\right)=A, \sup \left(J_{k}\right)=B$ ) and let

$$
h_{J_{i}}=1_{f \mid J_{i}}
$$

Then

$$
1_{f}=h_{J_{1}}+h_{J_{2}}+\cdots+h_{J_{k}} .
$$

Because the critical values are finite in number and distinct there is a neighborhood $U$ of $f(A)$ containing no other critical values. Thus $h_{J_{t}}$ is analytic in $U$ for $i=2,3, \ldots, k$. Now, $h_{J_{1}}$ has a non-pole discontinuity at $f(A)$ because: if $f^{\prime}(A)>0$ then

$$
\lim _{y \rightarrow f(A)^{+}} h_{J_{1}}(y)=\frac{1}{f^{\prime}(A)}, \quad \lim _{y \rightarrow f(A)^{-}} h_{J_{1}}(y)=0 .
$$


Similarly if $f^{\prime}(A)<0$, in which case

$$
\lim _{y \rightarrow f(A)^{-}} h_{J_{1}}(y)=\frac{-1}{f^{\prime}(A)}, \quad \lim _{y \rightarrow f(A)^{+}} h_{J_{1}}(y)=0 .
$$

Thus since $h_{J_{1}}$ has a non-pole discontinuity at $f(A)$ and the other $h_{J}$ 's are analytic near $f(A)$, it follows that $1_{f}$ has a non-pole discontinuity at $f(A)$. $f(B)$ is the other possible non-pole discontinuity of $1_{f}$, so we can find $f(A)$ or $f(B)$.

For simplicity assume $f^{\prime}(A)>0$, then we have for sufficiently small $\varepsilon>0$ that

$$
1_{f}=h_{J_{2}}+h_{J_{3}}+\cdots+h_{J_{k}} \text { on }(f(A)-\varepsilon, f(A))
$$

and

$$
1_{f}=h_{J_{1}}+h_{J_{2}}+\cdots+h_{J_{k}} \text { on }(f(A), f(A)+\varepsilon)
$$

let $G=$ analytic continuation of $1_{\left.f\right|_{(f(A)-\varepsilon, f(A))}}$ into $[f(A), f(A)+\varepsilon)$, then we have

$$
G=h_{J_{2}}+h_{J_{3}}+\cdots+h_{J_{k}} \text { on }(f(A)-\varepsilon, f(A)+\varepsilon) .
$$

Thus

$$
\left.1_{f}\right|_{(f(A), f(A)+\varepsilon)}-\left.G\right|_{(f(A), f(A)+\varepsilon)}=h_{\left.J_{1}\right|_{(f(A), f(A)+\varepsilon)}}
$$

Now $h_{\left.J_{1}\right|_{(f(A), f(A)+e)}}$ is known so we know $f$ near $A$ :

$$
\begin{aligned}
& \frac{d}{d y}\left(\left.f\right|_{J_{1}}\right)^{-1}=h_{J_{1}} \quad(\text { near } f(A)), \text { i.e. } \\
& \left(\left.f\right|_{J_{1}}\right)^{-1}=f(A)+\int_{f(A)}^{y} h_{J_{1}} d y \quad(\text { near } f(A)) .
\end{aligned}
$$

Therefore

$$
\left.f\right|_{J_{1}}=\left(f(A)+\int_{f(A)}^{y} h_{J_{1}} d y\right)^{-1} \quad(\text { near } A) .
$$

Since $f$ is analytic, knowing it near $A$, there is a unique analytic continuation to the rest of $[A, B]$.

The same argument holds if $f^{\prime}(A)<0$ or if $f^{\prime}(B) \neq 0: 1_{f}$ jumps up at $f(A)$ (or $f(B)$ ) and we may subtract the analytic continuation of "before the jump" from "after the jump" and the difference is exactly (a restriction of) $h_{J}$ where $J$ is the interval containing the endpoint $A(B)$. This being the derivative of $\left(\left.f\right|_{J}\right)^{-1}$ we can compute $\left.f\right|_{J}$ and then extend it to the rest of the domain by analytic continuation. 
Case 2. $f^{\prime}(A)=0, f^{\prime}(B)=0$. The argument is the same once we have singled out the poles $f(A)$ and $f(B)$ from the others; to do this requires some ideas:

If $y_{0}$ is a pole of $1_{f}$, since we assume non-degenerate and distinct critical values, $y_{0}$ is the value of a unique local maximum or minimum. It follows that the pole is one-sided i.e., if $y_{0}$ corresponds to a local maximum then

$$
\lim _{y \rightarrow y_{0}^{-}} 1_{f}(y)=\infty \text { and } \lim _{y \rightarrow y_{0}^{+}} 1_{f}(y)<\infty,
$$

or, if $y_{0}$ is the value of a local minimum then

$$
\lim _{y \rightarrow y_{0}^{+}} 1_{f}(y)=\infty \text { and } \lim _{y \rightarrow y_{0}^{-}} 1_{f}<\infty .
$$

For such a one-sided pole $y_{0}$, choose $\varepsilon>0$ so small that $\left(y_{0}-\varepsilon, y_{0}+\varepsilon\right)$ contains no other critical values of $f$.

Define.

$$
\varepsilon\left(y_{0}\right)= \begin{cases}\left(y_{0}-\varepsilon, y_{0}\right) & \text { if } y_{0} \text { corresponds to a minimum } \\ \left(y_{0}, y_{0}+\varepsilon\right) & \text { if } y_{0} \text { corresponds to a maximum }\end{cases}
$$

and

$$
\varepsilon^{\prime}\left(y_{0}\right)= \begin{cases}\left(y_{0}, y_{0}+\varepsilon\right) & \text { if } y_{0} \text { corresponds to a minimum } \\ \left(y_{0}-\varepsilon, y_{0}\right) & \text { if } y_{0} \text { corresponds to a maximum. }\end{cases}
$$

Thus $1_{f}$ has a pole in $\varepsilon^{\prime}\left(y_{0}\right)$ and none in $\varepsilon\left(y_{0}\right)$; also $1_{\left.f\right|_{e\left(y_{0}\right)}}$ has an analytic continuation into $\varepsilon^{\prime}\left(y_{0}\right)$ which is exactly $1_{\left.f\right|_{V}}$ where $V=[A, B] \backslash$ (a neighborhood of the critical point corresponding to $y_{0}$ ).

Define. The pole part of $1_{f}$ at $y_{0}$ is

$$
\pi_{y_{0}}(y)=1_{f_{\ell^{\prime}\left(y_{0}\right)}}-\left(\text { analytic continuation to } \varepsilon^{\prime}\left(y_{0}\right) \text { of }\left(1_{\left.f\right|_{\varepsilon\left(y_{0}\right)}}\right)\right. \text {. }
$$

This is defined for $y \in \varepsilon^{\prime}\left(y_{0}\right)$, and $\pi_{y_{0}}$ is the part of $1_{f}$ arising from $x$ 's near the critical point $x_{0}$ such that $f\left(x_{0}\right)=y_{0}$ (there are two such $x$ 's if $\left.x_{0} \notin\{A, B\}\right)$; the rest of $1_{f}$ is just the contribution from $x$ 's not near the critical point $x_{0}$; which contribution, being present on both sides of $y_{0}$ can be subtracted from $1_{f}$.

Each pole part arising from an interior critical point of $[A, B]$ consists of two terms: if $x_{0}$ is the interior critical point and if near $x_{0}$ we have $x_{-}$ and $x_{-}$as the two $x$ 's such that

$$
f\left(x_{ \pm}\right)=y
$$


then

$$
\pi_{y_{0}}(y)=\frac{1}{\left|f^{\prime}\left(x_{-}\right)\right|}+\frac{1}{\left|f^{\prime}\left(x_{+}\right)\right|} .
$$

These two terms are the branches of the pole part $\pi_{y_{0}}$. If $y_{0}=f(A)$ or $f(B)$, the pole part is also defined, but consists of just a single branch.

Define. A critical value $y_{1}$ is a successor of $y_{0}$ if the analytic continuation of $\pi_{y_{0}}$ has a pole at $y_{1}$ (i.e. if the maximal domain of definition of $\pi_{y_{0}}$ is the open interval between $y_{0}$ and $y_{1}$ ).

LEMMA 4. If $f^{\prime}(A)=0$ and $f^{\prime}(B)=0$, every critical value $y_{0}=f\left(x_{0}\right)$ has exactly one successor $f(x)$; between $x_{0}$ and $x$ there are no other critical points.

Proof. Consider the consecutive intervals on which $f$ is monotonic: $J_{i}$ and $J_{i+1}$, where $J_{i}=\left[x_{-}, x_{0}\right]$. Defining $\varepsilon^{\prime}\left(y_{0}\right)$ as before, we have for $y \in \varepsilon^{\prime}\left(y_{0}\right)$ such that

$$
\pi_{y_{0}}(y)=\frac{1}{\left|f^{\prime}\left(\left.f\right|_{J_{\imath}} ^{-1}(y)\right)\right|}+\frac{1}{\left|f^{\prime}\left(\left.f\right|_{J_{i+1}} ^{-1}(y)\right)\right|} .
$$

We can analytically continue $\pi_{y_{0}}$ by analytically continuing each of these two branches. The first is extended by $1_{\left.f\right|_{J_{t}}}$ and the second by $1_{\left.f\right|_{J_{t+1}}}$; thus the first extends to $f\left(x_{-}\right)$and the second to $f\left(x_{+}\right)$. Since $f\left(x_{-}\right) \stackrel{f}{\neq}\left(x_{+}\right)$ one is closer to $y_{0}=f\left(x_{0}\right)$ and $\pi_{y_{0}}$ extends analytically from $y_{0}$ to this nearer critical value. This proves the lemma: $f\left(x_{0}\right)$ has the successor $f\left(x_{-}\right)$or $f\left(x_{+}\right)$; and there are no other critical points between $x_{0}$ and $x=x_{ \pm}$.

One would hope that most critical values are successors and have a successor, and that $f(A)$ and $f(B)$ could be distinguished from the other poles as being the only critical values without both. Unfortunately, in some cases, a critical value is itself the successor of its successor; we still need more definitions.

Define A block of poles is an ordered sequence of poles of $1_{f}$ : $y_{0}, y_{1}, \ldots, y_{s}$ such that $y_{i} \neq y_{j}$ for $i \neq j$, such that for all $i$ either $y_{i}$ is the successor of $y_{i+1}$ or $y_{i+1}$ is the successor of $y_{i}$, and such that the sequence is maximal in the sense that it cannot be properly contained in any longer such sequence.

Our task is to discover which blocks of poles contain $f(A)$ and $f(B)$, and to single out those poles from the others of their respective blocks. 
Proposition 5. If $\pi_{y}$ and $\pi_{y_{i+1}}$ are the pole parts of successors then they may be analytically continued so as to share a branch.

Proof. In the notation of above

$$
\begin{aligned}
\pi_{y_{i}} & =\frac{1}{\left|f^{\prime}\left(\left.f\right|_{J_{i}} ^{-1}\right)\right|}+\frac{1}{\left|f^{\prime}\left(\left.f\right|_{J_{i+1}} ^{-1}\right)\right|} \\
\pi_{y_{i+1}} & =\frac{1}{\left|f^{\prime}\left(\left.f\right|_{J_{i+1}} ^{-1}\right)\right|}+\frac{1}{\left|f^{\prime}\left(\left.f\right|_{J_{i+2}} ^{-1}\right)\right|} .
\end{aligned}
$$

The second term of $\pi_{y_{i}}$ is the same as the first term of $\pi_{y_{i+1}}$, but defined in different domains: $\varepsilon^{\prime}\left(y_{i}\right)$ and $\varepsilon^{\prime}\left(y_{i+1}\right)$. Since $y_{i}$ and $y_{i+1}$ are in the relation of successors the maximal domain of extension of one pole part contains the maximal domain of extension of its successor. In the latter domain both pole parts can be defined and, there, they share the branch

$$
\frac{1}{\left|f^{\prime}\left(\left.f\right|_{J_{t+1}} ^{-1}\right)\right|} \text {. }
$$

In this domain into which both pole parts can be extended the difference

$$
\pi_{y_{i}}-\pi_{y_{t+1}}
$$

is defined and consists of the remaining branches of $\pi_{y_{i}}$ and $\pi_{y_{t+1}}$, the shared branch cancels.

Suppose, for example, that $y_{i}$ is an endpoint and $y_{i+1}$ is its successor, then the difference $\pi_{y_{t}}-\pi_{y_{i+1}}$ is defined in the maximal domain of extension of $\pi_{y_{i+1}}$ or at least it can be extended to that domain and consists only of the single unshared branch of $\pi_{y_{i+1}}$. This remaining branch has a maximal domain of extension with a pole corresponding to the value of $f$ at the third critical point counting in from the critical endpoint. If that pole is $y_{i+2}$ then the maximal domain of extension of $\pi_{y_{i}}-\pi_{y_{i+1}}$ contains the maximal domain of extension of $\pi_{y_{i+2}}$; in this latter domain they share a branch and in this domain

$$
\left(\pi_{y_{i}}-\pi_{y_{i+1}}\right)+\pi_{y_{i+2}}
$$

is defined and, again, consists of one remaining branch of $\pi_{y_{1+2}}$.

If we could order the poles of $1_{f}$ according to the order of their corresponding critical points $y_{0}, y_{1}, \ldots, y_{k}$. Then the alternating sum:

$$
\pi_{y_{0}}-\pi_{y_{1}}+\pi_{y_{2}} \cdots+(-1)^{k} \pi_{y_{k}}
$$

makes sense as follows: for each $i$

$$
\left(\pi_{y_{0}}-\pi_{y_{1}}+\cdots+(-1)^{i} \pi_{y_{\imath}}\right)
$$


has a maximal domain of extension which contains the maximal domain of extension of $\pi_{y_{i+1}}$. In that domain they share a branch, and so the difference

$$
\left(\pi_{y_{0}}-\pi_{y_{1}} \cdots+(-1)^{i} \pi_{y_{t}}\right)+(-1)^{i+1} \pi_{y_{l+1}}
$$

is defined in this domain; again the shared branch cancels.

Digression. We can distinguish the poles corresponding the local maxima from those corresponding to local minima (recall the definition of $\left.\varepsilon^{\prime}\left(y_{0}\right)\right)$ and can think of the sum as being

$$
\sum_{\max , s} \pi_{y_{t}}-\sum_{\text {min's }} \pi_{y_{j}} \text {. }
$$

It is an interesting observation that when we form this entire sum, all branches cancel and we are left with zero. The sum only makes sense however when the terms are added in order as before, in this order:

$$
\sum_{i}(-1)^{i} \pi_{y_{i}}=0 \text {. }
$$

This says no more than that the graph of the original function $f$ is connected, it is a topological statement. Given a distribution $\omega_{f}$ and its derivative $1_{f}$, we may ask: What are necessary and sufficient conditions that these arise from an analytic function $f$ with distinct critical values? At this point we can see that the supposed $1_{f}$ must have the appropriate kinds of one-sided poles but, more subtly, it must also have this property: that the alternating sum (in some order) of its pole parts is zero. Since functions which are $C^{1}$ and analytic between critical points also have such pole-parts with the alternating sum identically zero, this condition is not sufficient to guarantee that $f$ is globally analytic. However I believe it is sufficient if we consider such piecewise analytic functions. End Digression

Each block of poles $\left(z_{0}, z_{1}, \ldots, z_{s}\right)$ has two external poles $z_{0}$ and $z_{s}$. Amongst the external poles of all the blocks we must decide which correspond to an endpoint value $f(A)$ or $f(B)$. If $y_{i}$ is an external pole of a block (or any pole for that matter) there exists a sequence of poles $y_{0}$, $y_{1}, \ldots, y_{i-1}$ such that the alternating sum

$$
\pi_{y_{0}}-\pi_{y_{i}}+\cdots+(-1)^{i-1} \pi_{y_{i-1}}
$$

is defined and has an extension with a pole at $y_{i}$ and is "maximal" in the sense that the sequence $y_{0}, y_{1}, \ldots, y_{i-1}$ cannot be supplemented by including $y_{-k}, y_{-k+1}, \ldots, y_{-1}$ s that

$$
y_{-k}, \ldots, y_{-1}, y_{0}, y_{1}, \ldots, y_{i-1}
$$

still has this property. 
LEMMA 6. (a) If $y_{i}$ does not correspond to the value of an endpoint $\left(y_{i} \notin\{f(A), f(B)\}\right)$ then $y_{0}, y_{1}, \ldots, y_{i-1}$ may be chosen outside of the block containing $y_{i}$.

(b) If $y_{i} \in\{f(A), f(B)\}$ then $y_{0}, y_{1}, \ldots, y_{i-1}$ must be chosen using some of the poles of the block containing $y_{i}$.

Proof. If $y_{i}=f(A)$ or $f(B)$, it has a successor and is contained in some block containing more than just the critical value $y_{i}$. For a maximal sequence $y_{0}, y_{1}, \ldots, y_{i-1}$ such that

$$
\sum(-1)^{j} \pi_{y_{j}}
$$

is defined and has some domain extending only up to $y_{i}$, it is necessary that $y_{i-1}$ be in the block of $y_{i}$. If $y_{i}$ is not the value of an endpoint ( $A$ or $B)$, but is the external pole of some block, we can choose $y_{0}, y_{1}, \ldots, y_{i-1}$ not contained in the block of $y_{i}$.

Now we are ready to finish the proof of the Theorem. Lemma 6 tells us how to find $f(A)$ and $f(B)$. Say $y_{0}=f(A)$. Then setting

$$
\tilde{\pi}=\text { analytic continuation of } \pi_{y_{0}}
$$

we have

$$
\tilde{\pi}=\frac{d}{d y}\left(\left.f\right|_{[A, A+\varepsilon)} ^{-1}\right) \quad \text { near } y_{0}
$$

As before:

$$
\left.f\right|_{[A, A+\varepsilon)} ^{-1}(y)=f(A)+\int_{f(A)}^{y} \tilde{\pi}
$$

so

$$
\left.f\right|_{[A, A+\varepsilon)}=\left(f(A)+\int_{f(A)}^{y} \tilde{\pi}\right)^{-1} .
$$

Thus, $f$ is determined on $[A, B]$ by analytically continuing $\left.f\right|_{[A, A+\varepsilon)}$.

\section{REFERENCES}

[1] W. F. Pohl, Some integral formulas for space curves and their generalizations, Amer. J. Math., V. XC, No. 4 (Oct. 1968), p. 1328.

Received June 25, 1985. 



\section{PACIFIC JOURNAL OF MATHEMATICS EDITORS}

\author{
V. S. VARADARAJAN \\ (Managing Editor) \\ University of California \\ Los Angeles, CA 90024 \\ Herbert Clemens \\ University of Utah \\ Salt Lake City, UT 84112 \\ R. FINN \\ Stanford University \\ Stanford, CA 94305
}

\section{HERMANN FLASCHKA}

University of Arizona

Tucson, AZ 85721

RAMESH A. GANGOLLI

University of Washington

Seattle, WA 98195

VAUGHAN F. R. JONES

University of California

Berkeley, CA 94720

ROBION KIRBY

University of California

Berkeley, CA 94720
C. C. MOORE

University of California

Berkeley, CA 94720

H. SAMELSON

Stanford University

Stanford, CA 94305

HAROLD STARK

University of California, San Diego

La Jolla, CA 92093

\section{ASSOCIATE EDITORS}
R. ARENS
E. F. BECKENBACH
B. H. NEUMANN
F. WOLF
K. YOSHIDA
(1906-1982)

\section{SUPPORTING INSTITUTIONS}

UNIVERSITY OF ARIZONA UNIVERSITY OF OREGON

UNIVERSITY OF BRITISH COLUMBIA UNIVERSITY OF SOUTHERN CALIFORNIA

CALIFORNIA INSTITUTE OF TECHNOLOGY STANFORD UNIVERSITY

UNIVERSITY OF CALIFORNIA

MONTANA STATE UNIVERSITY

UNIVERSITY OF HAWAII

UNIVERSITY OF NEVADA, RENO

UNIVERSITY OF TOKYO

NEW MEXICO STATE UNIVERSITY

UNIVERSITY OF UTAH

OREGON STATE UNIVERSITY

WASHINGTON STATE UNIVERSITY

UNIVERSITY OF WASHINGTON 


\section{Pacific Journal of Mathematics}

\section{Vol. 126, No. $1 \quad$ November, 1987}

John Dauns, Uniform dimensions and subdirect products $\ldots \ldots \ldots \ldots \ldots 1$

William B. Jacob, Quadratic forms over dyadic valued fields. I. The graded

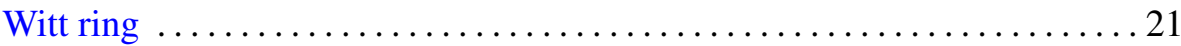

Michael R. Kelly, Minimizing the number of fixed points for self-maps of

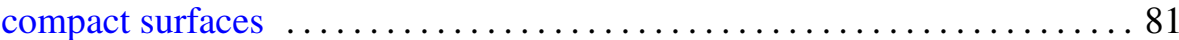

Edward Kissin, On some reflexive operator algebras constructed from two sets of closed operators and from a set of reflexive operator algebras . . 125

Robert Langlands, The Dirac monopole and induced representations . . . . . 145

David A. Stegenga and Kenneth R. Stephenson, Generic covering properties for spaces of analytic functions. II $\ldots \ldots \ldots \ldots \ldots \ldots \ldots 153$

Gerard Alan Venema, Approximating codimension two embeddings of cells 165

Peter Waksman, Determining an analytic function from its distribution of values 